\title{
An electrostatic precipitator system for the Martian environment
}

\author{
C.I. Calle ${ }^{1}$, P.J. Mackey ${ }^{1}$, M.D. Hogue ${ }^{1}$, M.R. Johansen ${ }^{1}$, J.D.Kelley ${ }^{2}$, J.R. Phillips III $^{2}$, \\ and J.S. Clements ${ }^{2}$
}

${ }^{1}$ Electrostatics and Surface Physics Laboratory, NASA, Kennedy Space Center, FL 32899; e-mail: carlos.i.calle@nasa.gov

2 Department of Physics and Astronomy, Appalachian State University, Boone, NC 28608, USA

\begin{abstract}
Human exploration missions to Mars will require the development of technologies for the utilization of the planet's own resources for the production of commodities. However, the Martian atmosphere contains large amounts of dust. The extraction of commodities from this atmosphere requires prior removal of this dust. We report on our development of an electrostatic precipitator able to collect Martian simulated dust particles in atmospheric conditions approaching those of Mars. Extensive experiments with an initial prototype in a simulated Martian atmosphere showed efficiencies of $99 \%$. The design of a second prototype with aerosolized Martian simulated dust in a flow-through is described.
\end{abstract}

Keywords: Space applications, electrostatic precipitator, particle control, particle charging

\section{Introduction}

The human exploration of Mars will require the utilization of the planet's resources for the production of consumables and for the construction, manufacturing, and repair of space utilities and power [1]. The extraction of commodities such as oxygen, methane, and water from the Martian atmosphere will require the removal of atmospheric dust from the intakes of the processing chambers. Filtering the dust will require upstream compression in the low pressure of the Martian atmosphere (7 to 10 mbars), which is difficult to achieve. Dust removal by electrostatic precipitation, an efficient and mature technology on Earth, is also challenging on Mars due to the limited electrostatic potential differences achievable in the low pressure atmospheric environment of Mars.

We report on our development of a static electrostatic precipitator design capable of removing dust particles in the limited electrostatic fields of a simulated Martian environment with removal efficiencies reaching $99 \%$. We also discuss the design of a similar precipitator in a flow for a one-tenth-scale oxygen production demonstration reactor for a Mars mission.

\section{The Relevant Martian Atmospheric Environment}

The Martian atmosphere contains dust uplifted from the surface by dust devils and dust storms. Dust particles 2 to $4 \mu \mathrm{m}$ in diameter are constantly present in the atmosphere.

\footnotetext{
${ }^{1}$ To whom any correspondence should be addressed.
} 
During dust devil and dust storm activity, particles up to $10 \mu \mathrm{m}$ in diameter are also present in the atmosphere. Larger particles, with diameters greater than $80 \mu \mathrm{m}$, are airlifted close to the surface and for short distances due to the saltation process [2]. Reactors for the extraction of commodities on Mars will likely be designed away from the surface to eliminate dealing with the larger saltating particles. Dust removal for these reactors will be concerned with particles $10 \mu \mathrm{m}$ and under in diameter.

Terrestrial electrostatic precipitators operate at electrostatic potentials in the $30-70 \mathrm{kV}$ range. These potentials are not possible in the low pressure Martian atmosphere, where electrical breakdown occurs at relatively low potentials. Electrical breakdown between two electrodes in a gas, known as Townsend breakdown [3], depends on the product of the gap $d$ between the electrodes and the gas number density. This relationship is called Paschen's law [4]. Historically, the gas pressure $p$ is used instead of the number density.

We performed experiments with a premixed gas to simulate the Martian atmosphere, composed of a mixture of $95 \% \mathrm{CO}_{2}, 2.7 \% \mathrm{~N}_{2}, 1.6 \% \mathrm{~A}, 0.13 \% \mathrm{O}_{2}$, and $0.07 \% \mathrm{CO}$ [5]. Data was taken at pressures ranging from 0.27 mbars to 466 mbars ( 0.2 torr to 350 torr) with gaps of $5 \mathrm{~mm}, 7.5 \mathrm{~mm}$, and $10 \mathrm{~mm}$. The low pressure data (Fig. 1) shows that breakdown potentials in $\mathrm{CO}_{2}$ and in the Martian gas mixture are extremely similar. It appears as if $\mathrm{CO}_{2}$ dictates the Paschen breakdown and that the other gases, present in relatively low concentrations, do not affect it substantially. The Martian gas breakdown voltages are equal to or slightly smaller than the $\mathrm{CO}_{2}$ voltages by an average of 15 volts, perhaps due to the known lower breakdown potentials of nitrogen and argon, present in small concentrations. Based on these results, we used a $95 \% \mathrm{CO}_{2} / 5 \%$ humid air mixture to approximate the Martian atmosphere for this study as well as a $100 \% \mathrm{CO}_{2}$ atmosphere.

\section{Experiments and Results}

A typical electrostatic precipitator consists of two evenly spaced electrodes with a potential difference that generates an electric field able to drive charged dust particles to one of the electrodes for collection. In a single-stage precipitator, the electric field is set up to generate a corona that charges the dust particles. Since this field also drives the charged particles, increasing the field strength will increase the particle collection. The value of the electrical breakdown for the electrode configuration, gas composition, and pressure determines the maximum value of the electric field. In the case of Mars, parallel plate electrodes separated by a $10 \mathrm{~cm}$ gap will breakdown at around $3 \mathrm{kV}$, a value much smaller than on Earth, which allows voltages up to about $300 \mathrm{kV}$ for the same configuration. 


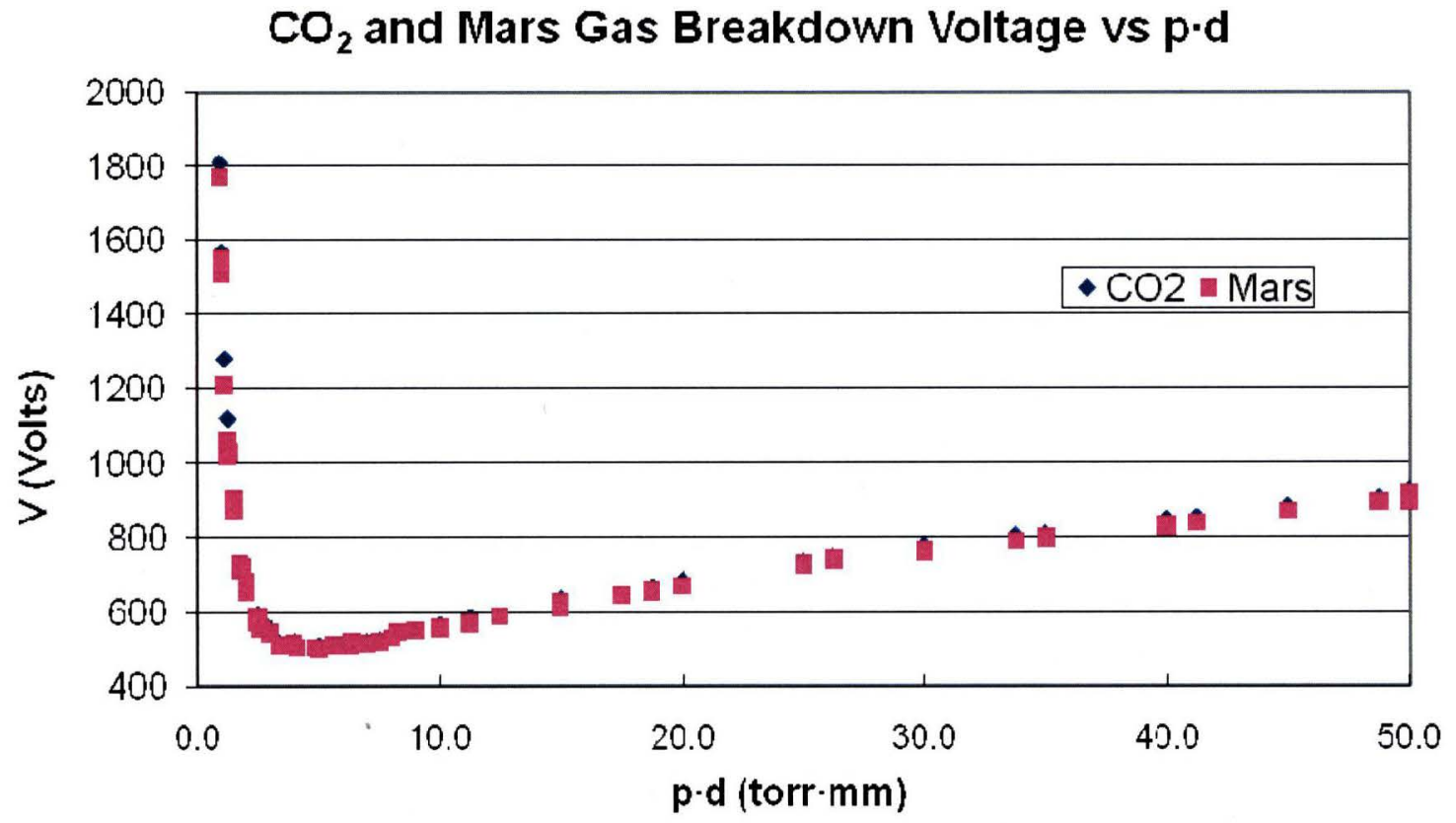

Figure 1. Paschen breakdown potentials versus pressure $\times$ distance for a Martian gas mixture (red squares) and for $\mathrm{CO} 2$ (blue triangles) [5].

A prototype cylindrical precipitator was designed and built with seven different diameter configurations [Calle et al 2011]. Experiments were performed with the configurations with the best $I-V$ curves $(30 \mathrm{~cm}$ long, $7.1 \mathrm{~cm}$ in diameter outer cylinder with $0.3 \mathrm{~cm}$ and $70 \mu \mathrm{m}$ inner electrodes, respectively). About $5 \mathrm{mg}$ of dust with properties similar to Martian dust were introduced into a vacuum chamber evacuated to a simulated Martian atmosphere of 9 mbars of carbon dioxide. The Martian simulated dust was aerosolized in the chamber and allowed to fall through the vertically mounted precipitator. Collection efficiencies of 94 to $96 \%$ were achieved with these configurations [Calle et al 2011].

Experiments with a cylindrical precipitator $45 \mathrm{~cm}$ long, $7.1 \mathrm{~cm}$ in diameter outer cylinder and a $100 \mu \mathrm{m}$ inner electrode were also performed. As before, ten runs with $5 \mathrm{mg}$ of Martian simulated dust that were aerosolized in the chamber were performed. The high voltage electrode was set at a potential of positive $1.6 \mathrm{kV} \mathrm{DC}$ and the outer cylinder was electrically grounded. Whatman 542 Hardened Ashless filter paper was used to collect the dust that was not precipitated. The same type paper was used after the experiment was completed to remove the dust that was collected in the cylinder. After burning the paper at $550^{\circ}$ Celsius, collection efficiencies were obtained by weighing the crucibles containing the dust. Efficiencies of 98 and $99 \%$ were obtained, thus approaching the efficiencies of terrestrial precipitators.

In the experiments just described, the dust passed through the precipitator mainly under the action of the gravitational force. A commodity producing reactor to be deployed on Mars will need to remove Martian atmospheric dust from the gas stream entering the reactor. Current designs call for a flow of $88 \mathrm{~g} / \mathrm{hr}$ or 0.74 standard liters per minute (SLPM), which correspond to about 94 liters per minute at 8 mbars of pressure. For a planned onetenth-scale oxygen production demonstration reactor, this flow rate will be $9.4 \mathrm{~L} / \mathrm{min}$. 
We have designed and built a prototype precipitator in a flow through system (Fig. 2). The cylindrical design uses a $6.985 \mathrm{~cm}$ diameter, $91.44 \mathrm{~cm}$ length collector and a $100 \mu \mathrm{m}$ diameter high voltage electrode. Two laser particle counters provide dust particle counts before and after entering the electric field collecting region. The precipitator is outfitted with a vacuum rated feed-throughs and is evacuated to Martian pressures with a vacuum pump. A removable mechanical feed-through contains a dust holding pan. Dust is introduced prior to evacuating the precipitator.

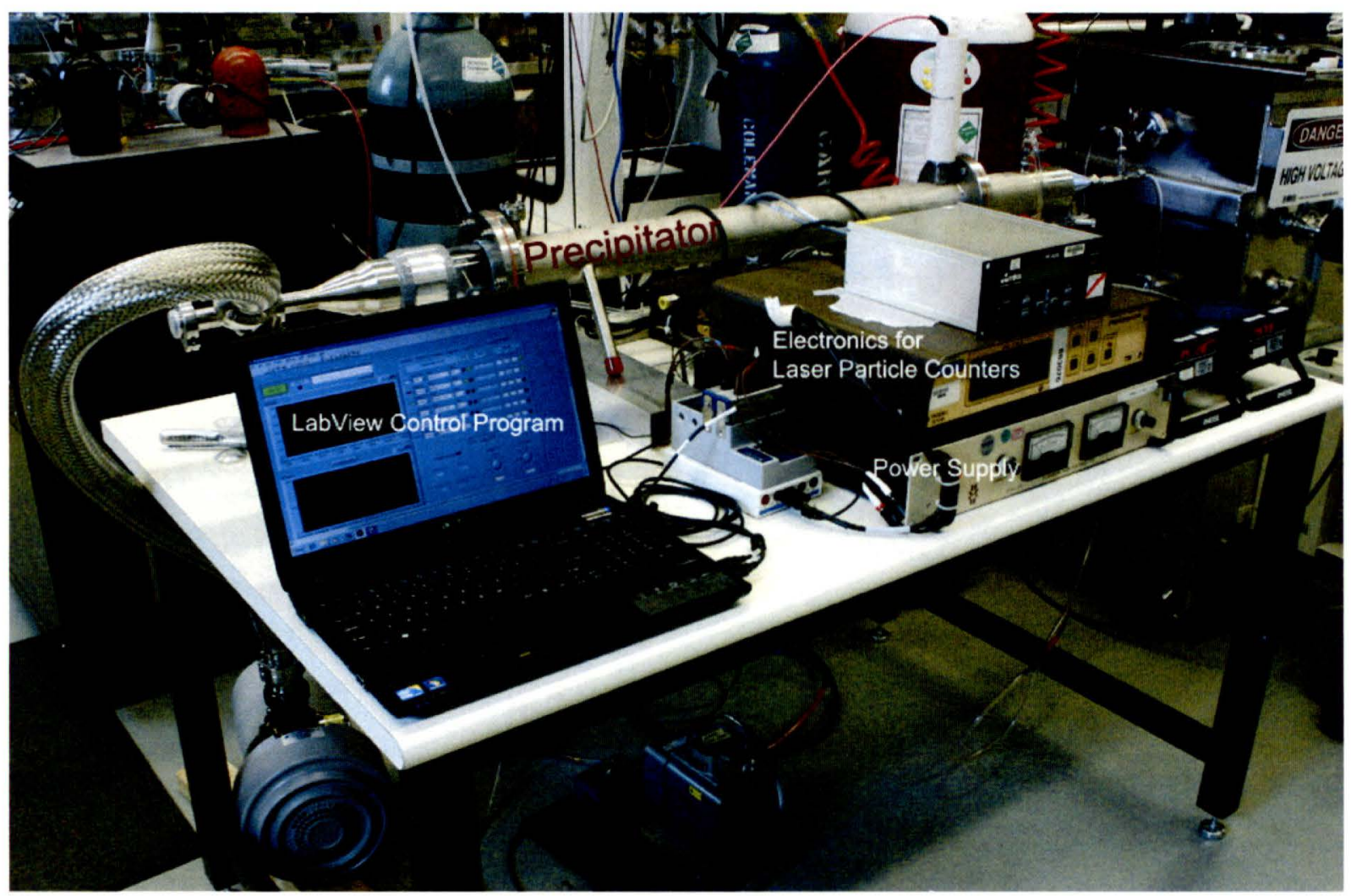

Figure 2. Electrostatic precipitator in a flow through configuration.

Current-Voltage (I-V) curves were obtained for static 9 mbar pressure conditions in air and in $\mathrm{CO}_{2}$ (Fig. 3). The applied voltage was started at $100 \mathrm{~V}$ and increased in increments of $50 \mathrm{~V}$ until the corona current reached about $500 \mu \mathrm{A}$. This current is larger than the 250 $\mu \mathrm{A}$ obtained with the earlier static design due to the increased length of the new precipitator. 


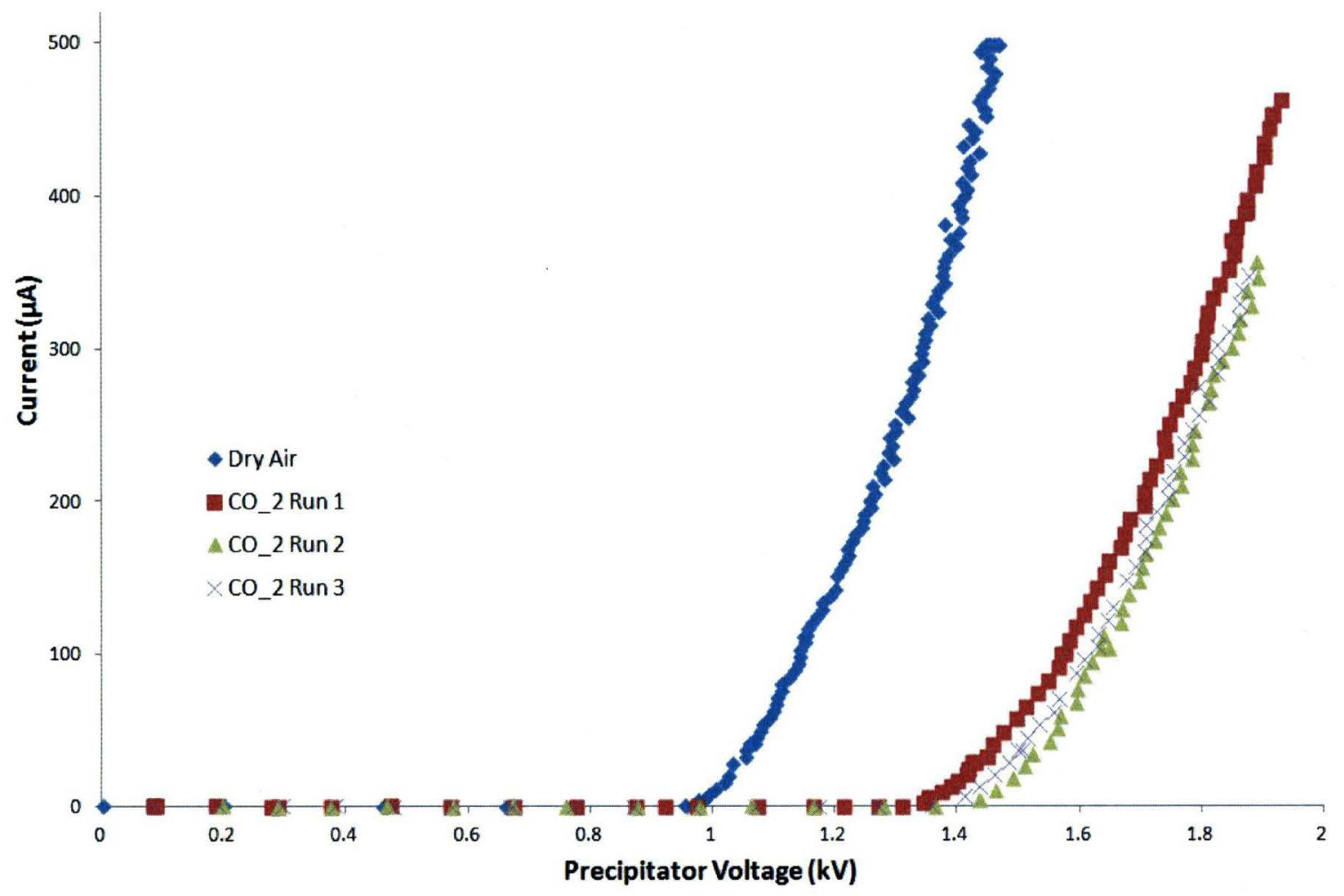

Figure 3. Current-voltage curves for the precipitator in a flow through configuration under 9 mbar no flow conditions.

Initial particle collection experiments with the precipitator were then performed after introducing Martian simulated dust with the mechanical feed-through. Although the special dust used contained particle sizes as large as $10 \mu \mathrm{m}$, the laser particle counter showed that only particles $5 \mu \mathrm{m}$ and under were actually aerosolized. We think that the larger mass of the bigger particles outweighed the drag force and gas momentum at 9 mbars of pressure, thus preventing them from being aerosolized.

We were able to complete one single run of our experiment before disassembling the precipitator for a planned move of the laboratory to a new location. This initial run showed significant counts on the upstream and downstream laser particle counters with the high voltage turned off. Once the high voltage was turned on, the downstream counts all but disappeared, providing encouraging results. Additional experiments are currently being planned once the laboratory is in operation again so that meaningful collection efficiency data can be reported.

\section{References}

[1] Sanders, G.B. et al, "Results from the NASA Capability Roadmap Team for In-Situ Resource Utilization (ISRU)," in 2005 International Lunar Conference, Toronto, Canada

[2] Landis G A, Herkenhoff K, Greeley R, Thompon S, Whelley P and the MER Athena Science Team 2006 Dust and sand deposition on the MER solar arrays as viewed by the Microscopic Imager LPS 371937

[3] Townsend, J. S., Electricity in Gases, New York: Oxford University Press, 1914 
[4] Paschen, F., Wied. Ann., vol. 37, p. 69, 1889

[5] Calle, C.I., J. S. Clements, J. Willis, and C. R. Buhler, "Paschen Breakdown Experiments in a Martian Atmosphere," NASA Technical Memorandum 2004211535, pp.86-87, 2004 